\title{
Fluid Dynamics Properties of Barium Hexaferrite Particle
}

\author{
Perdamean Sebayang ${ }^{1)}$, Muljadi $^{1)}$, Anggito Tetuko ${ }^{1)}$, Priyo Sardjono ${ }^{1)}$ \\ ${ }^{1)}$ Pusat Penelitian Fisika LIPI \\ Komplek Puspiptek Gedung 440, Tangerang Selatan, 15314 \\ Telp : (021) 7560570, Fax : (021) 7560554 \\ E-mail: pard003@lipi.go.id; perdameans@yahoo.com
}

\begin{abstract}
Particle size distribution of Barium Hexaferrite sample has been performed with commonly used methods of mathematical models by Rosin-Rammler (RR model) distribution. By using sieving method from 20-400 mesh, the basis of network analysis distribution function $F(d)$ and density function, $f(d)$ were obtained. Particle size estimation was performed using sedimentation gravitation based on Stokes law to obtained Reynolds numbers and terminal velocity of flocs in medium value has been calculated. The results of Reynolds numbers shows that Barium hexaferrite flocs in ethanol medium in laminar flow, whereas terminal velocity increases as larger particle size and density, however, bulk density reduce due to contained highly porous in the sample which yields lower bulk density. The relationship of turbidity with the floc size has been evaluated. The results show that turbidity and bulk density increases as smaller particle size, meanwhile, terminal velocity reduced. Differences in turbidity for each sample (20-400 mesh) has been determined which shows two region instead, with first region from 150-850 $\mu$ m yields larger differences compared to the second region: 37-105 $\mu \mathrm{m}$.
\end{abstract}

Keywords: Rosin-Rammler model, terminal velocity, Reynolds numbers, turbidity, sedimentation

\section{INTRODUCTION}

Particle size is one of the most important physical properties of solids, which are used in many fields of human activity, such as construction, waste management, metallurgy, fuel fabrication, etc. The aggregation of fine particles and colloids into larger assemblages is a process that resulting aggregates that form are known as 'flocculation' which are described as being highly porous, irregularly structured and loosely connected aggregates composed of smaller primary particles [1]. Once the solid particles are produced, they may be separated from water using sedimentation, flotation, filtration and thickening techniques [2]. The physical characteristics of floc are therefore fundamental in determining their removal efficiency. For example, large compact flocs have a high settling rate that results in a treated water of low turbidity during settlement [3], whilst large and porous flocs aid filtration due to high permeability [4]. A number of methods aimed at determining Particle Size Distribution (PSD) (i.e., sieving, cycloning, microscopy, etc.), where sieving are the known as the most common method due to its simplicity, which have been described in the literature [5]. There are several analysis techniques, both mathematical models GGS and RR distribution has widely used to study the PSD of solids, which have been applied to a cork granulate sample. However, based on literature [6-9], RR model were used to provide excellent results when applied to the sample of agglomerate cork studied, which leads to a more accurate separation of the different particle sizes to a better industrial profit of the material. Hence, with analysis technique were used will depend on the ultimate goal of the characterization. The aim of this study is to obtain the distribution $\mathrm{F}(\mathrm{d})$ (mass fraction) and density $\mathrm{f}(\mathrm{d})$ functions (number of particles binned between two given mesh sizes) of a Barium Hexaferrite particles by applying the RR distribution of mathematical models to the PSD data obtained by sieving through different size meshes [10]. The results of PSD analysis can be expressed according to the particle diameter indicating the nominal mesh sizes, or by particle size distribution, in grams, in percentage by weight of each fraction (differential distribution, as the cumulative percentage of sizes below a given value, undersize, and as the cumulative percentage of size above a given value, oversize) [11]. In this experiment, settling techniques was used to perform the gravity sedimentation. This technique is one of sedimentation method to determine floc structural 
characteristics, which takes on an extra relevance because the settling behavior of aggregates is an important parameter for optimizing the sedimentation process. Floc settling behavior is dependent upon size, effective density and porosity [11]. Settling velocity has several advantages such as measuring fractals of compact flocs, cheap and simple method, and also good for aggregates particles which made from a number of different primary particles [12]. Turbidity measurement was conducted and to observed the sedimentation as theoretically with larger particle size will yields a lower turbidity value.

\section{METHODOLOGY}

Bulk green-body barium hexaferrite from PT. Neomax, Cilegon were used as in the form of powder. A 100 gram of bulk barium hexaferrite was which was firstly grinded by using mortar and sieves using standard sieves of known apertures with different mesh size from 20, 30, 40, 50, 100, 150, 200, and 400 meshes. To determine the particle distribution of barium hexaferrite by using mathematical RR model distribution. The function $\mathrm{F}(\mathrm{d})$ is particularly suited to represent powders made by grinding, milling, and crushing operations [13]. The general expression of the RR model is given by:

$$
F(d)=1-\exp \left[\left(-\frac{d}{i}\right)\right]^{m}
$$

Where, $\mathrm{F}(\mathrm{d})$ is a distribution function, $d$ particle size (diameter of spherical particles) in $\mu \mathrm{m}, \quad l$ is mean particle size $(\mu \mathrm{m})$, and $m$ is the measured of the spread of particle sizes. Parameters $l$ and $m$ are adjustable parameters characteristic for the distribution. Eq. (1) can be rewritten as:

$$
\ln [-\ln [1-\mathrm{F}(\mathrm{d})]]=m \mathrm{x} \ln e t-\mathrm{m} \mathrm{x} \ln l
$$

By using the RR mathematical model for particle size distribution, a plot of the first term of Eq. 2, indicating the applicability of RR model for particle size distribution curve. For the regression analysis method of least squares is also frequently used to estimate the data point between two mesh sieves size. The correlation coefficient is used as a parameter indicating the relevance of the measured data set. The density function in RR mathematics model will be:

$$
f(d)=\frac{m}{l^{m}} x d^{m-1} \exp \left[\left(-\frac{d}{l}\right)\right]^{m}
$$

Particle size distribution analysis was done by plotting the graph of cumulative \% passing, distribution function and density function against particle size using equation (1) and (3), whereas for the fitting to RR model by using equation (2). From the grinded powder Barium Hexaferrite and filtered using mesh sieves and each of which passing powder (undersize) through sieves were then weight to calculate the density of the powder using pycnometer with ethanol (medium) and the others mixed in the toluene (medium) as dispersant to calculate bulk density of powder and settling velocity as well as particle flow through the medium, respectively. To determine the behavior of particle settling velocity in a medium were used sedimentation method (gravity sedimentation). A mathematical model was used to explain the phenomena of gravity sedimentation by using Stokes law and also Reynolds number can be obtained. Settling velocity can be applicable for spherical particles (assumed) falling through a medium not particle that could react to the medium.

To estimate the true value of terminal velocity, $\mathrm{V}_{\mathrm{T}}$, thus drag force must be taken account. For spherical particle under streamline condition (also known as laminar or Newtonian conditions), Stokes has determined drag force, $F_{D}=6 \pi r \mu V_{T}$. The ratio of inertia force divided by viscous force is known as Reynolds number (Re). The streamline condition can be determined when the fluid flowing past the streamline (laminar), $\operatorname{Re}<2100$, transitional stage with Re between 2100 and 4000 but when the flow is turbulent $\mathrm{Re}$ is greater than 4000 . During settling of a particle under streamline condition, force acting on the particle due to gravitational acceleration is given by: $\mathrm{mg}=\mathrm{V}\left(\rho_{\mathrm{s}}-\rho_{\mathrm{f}}\right) \mathrm{g}$, where $\mathrm{V}$ is the volume of spherical particles. In practice it is difficult to ascertain whether the flow past a falling particle is streamline or turbulent. Considering projected area, A, of a particle falling freely as $\mathrm{F}_{\mathrm{D}}$ as total drag force on particle, where constant $\mathrm{k}$ is a function of the shape of the particle and $\rho_{\mathrm{s}}$ is the density of the Barium hexaferrite powder, shown as:

$$
\mathrm{F}_{\mathrm{D}} \frac{\mathrm{nd}}{4}=\left(\rho_{\mathrm{g}}-\rho_{f}\right) \mathrm{g} k \mathrm{~d}_{\mathrm{p}}^{\mathrm{g}}
$$

Next, by dividing both sides of the equation by term $\rho_{\mathrm{F}} \mathrm{V}^{2}$ and multiplying by $\mathrm{Re}^{2}$, yields to below Eq. (6):

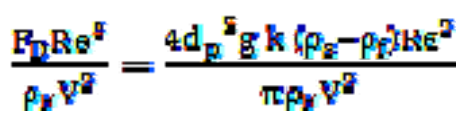


and by substituting $\mathrm{Re}=\mathrm{d}_{\mathrm{p}} \mathrm{V} \rho_{\mathrm{F}} / \mu$ and simplifying the equation,

$$
\frac{\mathrm{F}_{\mathrm{D}} \mathrm{R} \mathrm{e}^{\mathrm{z}}}{\rho_{\mathrm{F}} \mathrm{v}^{\mathrm{z}}}=\frac{4 \mathrm{~d}_{\mathrm{p}}{ }^{\mathrm{a}} \mathrm{gk}\left(\rho_{\mathrm{s}}-\rho_{\mathrm{f}}\right) \mathrm{PF}_{\mathrm{F}}}{\pi \mu^{2}}
$$

The right hand side of Eq. (5) deals with fluid properties only and diameter of particle falling under any condition can be computed by equating this value of left hand side Eq. (6) to determine Re in logarithmic form. Thus, from Table 1 and Table 2 from literature [13] as the correction value for the $\mathrm{Re}$, where the logarithmic function written as: $\mathrm{f}\left(\mathrm{Re}^{2}\right)=\log \left[\left(\mathrm{F}_{\mathrm{D}} / \rho_{\mathrm{f}} \mathrm{AV}_{\mathrm{T}}{ }^{2}\right) \mathrm{Re}^{2}\right]$ and $\log$ Re. Similarly, terminal velocity can be calculated from equation $\mathrm{V}$ $=\operatorname{Re} \mu / \mathrm{d}_{\mathrm{p}} \rho_{\mathrm{f}}$ (unit $\mathrm{m} / \mathrm{s}$ ).

Turbidity measurement has been done by using turbidity meter TU-2016 (calibrated using 0 and $100 \mathrm{NTU}$ as sample references) with sample consist of each undersize particle pass through the sieves 20-400 mesh, weight and mixed in toluene as medium. Turbidity data was taken for 400 data with interval $10 \mathrm{~s}$ for each data point.

\section{RESULTS AND DISCUSSIONS}

The sample powder from different particle sizes of grinded Barium Hexaferrite pass through each sieves was weigh to obtained cumulative mass \% passing as shown in Table 1. Particle size distribution analysis of barium hexaferrite powder as shown in the Fig. 1, cumulative passing (\%) against particle size which shows the spread of particle value denote as $\mathrm{m}$, the slope of the graph 0.2683 from Eq. (2) in logarithmic equation with Rsquared equal to 0.9143. From the fitting of measured data of Fig. 1, shows the logarithmic regression equation, the slope of the graph, $\mathrm{m}$ obtained and were used to calculate the distribution function of particle size Barium Hexaferrite in the sample using the given Eq. (1) with mean particle size, $l$, equal to $460 \mu \mathrm{m}$.

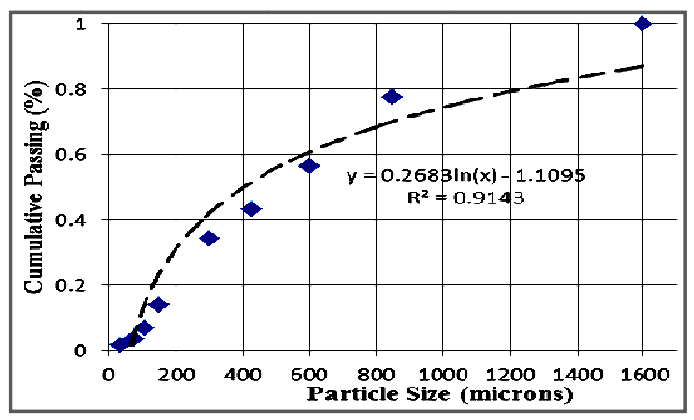

Figure 1. Plot logarithmic PSD curve obtained by sieving of cumulative $\%$ passing (undersize).
Rosin-Rammler distribution function, $F(d)$, shown in Fig. 2a. This function may represent the fraction by volume, mass, or by number of particles (flocs) with $\mathrm{R}^{2}$ equal to 0.9994 , this value represent that the point was perfectly fit the logarithmic curve. The value of the function at a given point is the fraction of the number of particles (mass or volume) that is below a given size.

Thus, the area under the curve between two sizes (i.e., $\mathrm{d}_{1}$ and $\mathrm{d}_{2}$ ) indicates the number of particles (expressed as particle mass or volume) whose diameters are comprised in this interval:

$$
F\left(d_{2}\right)-F\left(d_{1}\right)=\int_{d_{1}}^{d_{2}} f(d) d(d)
$$

Tabel 1. Size analysis with cumulative masses and fit of tested material to RR model.

\begin{tabular}{cccccc}
\hline $\begin{array}{c}\mathrm{d}, \\
\mu \mathrm{m}\end{array}$ & $\begin{array}{c}\text { Cum. } \\
\text { Mass \% } \\
\text { passing }\end{array}$ & $\mathrm{F}(\mathrm{d})$ & $\begin{array}{c}\mathrm{f}(\mathrm{d}) \\
\left(\mathrm{x} 10^{-3}\right)\end{array}$ & $\mathrm{y}$ & $\ln (\mathrm{d})$ \\
\hline 1600 & 1.00 & 0.753 & 0.058 & 0.335 & 0.058 \\
850 & 0.78 & 0.692 & 0.114 & 0.165 & 0.114 \\
600 & 0.56 & 0.658 & 0.164 & 0.071 & 0.164 \\
425 & 0.43 & 0.624 & 0.232 & -0.021 & 0.232 \\
300 & 0.34 & 0.590 & 0.327 & -0.115 & 0.327 \\
150 & 0.14 & 0.523 & 0.632 & -0.301 & 0.632 \\
105 & 0.07 & 0.490 & 0.877 & -0.396 & 0.877 \\
74 & 0.03 & 0.458 & 1.204 & -0.490 & 1.204 \\
37 & 0.02 & 0.392 & 2.386 & -0.699 & 2.386 \\
Pan & 0.00 & - & - & - & - \\
\hline
\end{tabular}

Where: $\mathrm{d}=$ aperture mesh size,

$$
\mathrm{y}=\ln \{-\ln [1-\mathrm{F}(d)]\}
$$

The inclination of distribution function (depending on the diameter of particles, $f(d)$, at each point indicates the density function $f(d)$, which is defined by Eq. 3 and shown in Fig. 2 b. This function represents the logarithmic curve, which corresponds to the proportion of particles of a certain size. From the Tabel 1, shows the value of distribution function, $F(d)$, thus it was possible by using Eq. (2) to calculate $\ln \{-\ln [1-\mathrm{F}(\mathrm{d})]\}$ and $\ln d$, by plotting both value to the graph as shown in the Fig. 3a. From the graph shows perfectly fits of experimental data to Rosin-Rammler models with $\mathrm{R}$-squared equal to 1 , thus the corresponding linear correlation coefficient between $\ln \{-\ln [1-\mathrm{F}(\mathrm{d})]\}$ and $\ln d$. The application of these expressions to the PSD curve allows one to extrapolate (for estimation purpose). Therefore from Fig. 3a, has been obtained 
the linear regression equation, $y=0.2683 x-1.6449$, can be used to estimate the distribution of particles in sample for particle size range within $37-1600$ $\mu \mathrm{m}$.

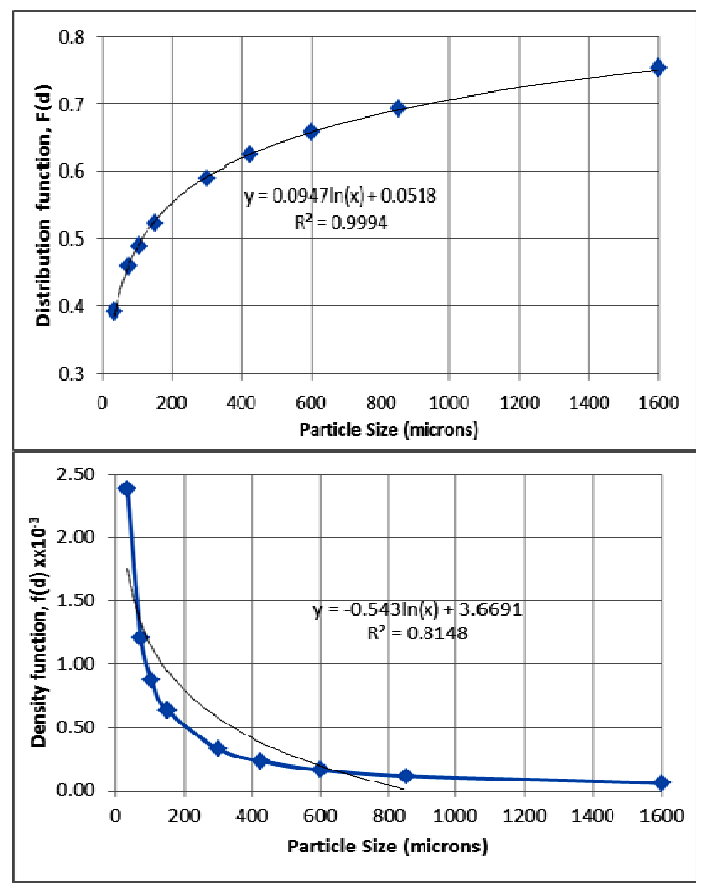

Figure 2. Plot of the Rosin-Rammler (a) Distribution function and (b) Density function against particle size.

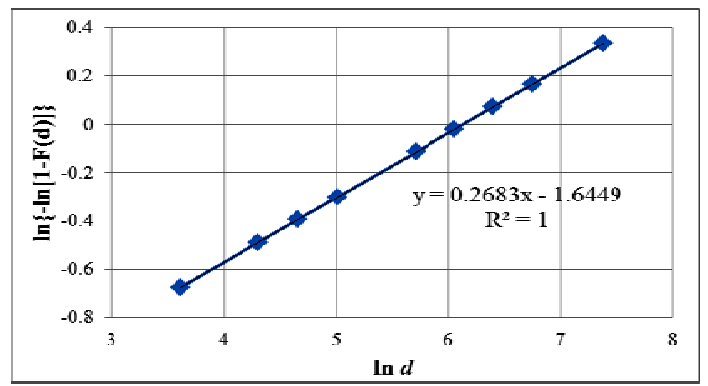

Figure 3. Plot of fitting curve experimental method to RR model.

Bulk density of grinded Barium Hexaferrite passed through each sieves (undersize) was determined by using pycnometer with ethanol medium. From the graph of bulk density against particle size in Fig. 4, shows the linear relationship between particle size with increasing value of bulk density of Barium Hexaferrite, where R-squared equal to 0.9664 . Therefore, linear equation, $y=-$
$0.6481 x+5722.4$ obtained from Fig. 4 , can be used for bulk density of Barium Hexaferrite as an estimation from certain particle size within 37-850 $\mu \mathrm{m}$.

Table 2. Based on Stokes law to determine terminal velocity and Reynold numbers.

\begin{tabular}{ccrc}
\hline $\begin{array}{c}\text { Aperture } \\
\text { size, } \mathrm{d} \\
(\mu \mathrm{m})\end{array}$ & $\begin{array}{c}\text { Bulk } \\
\text { density, } \\
\rho_{\mathrm{p}}\left(\mathrm{kg} / \mathrm{m}^{3}\right)\end{array}$ & Re & $\begin{array}{c}\text { Terminal } \\
\text { velocity, Vt } \\
\left(\mathrm{x} 10^{-3} \mathrm{~m} / \mathrm{s}\right)\end{array}$ \\
\hline 850 & 5204.98 & 247.70 & 30.79 \\
600 & 5265.56 & 125.03 & 21.77 \\
425 & 5481.75 & 71.61 & 16.91 \\
300 & 5504.65 & 33.88 & 11.28 \\
150 & 5651.03 & 7.50 & 4.87 \\
105 & 5667.58 & 3.64 & 3.36 \\
74 & 5673.44 & 1.37 & 1.79 \\
37 & 5683.70 & 0.05 & 0.13 \\
\hline
\end{tabular}

Additionally, larger particle size contained highly porous which yields lower bulk density. By using Eq. (6), to determine Reynold number and terminal velocity of particles (flocs) due to the gravitation sedimentation by assuming Barium Hexaferrite particle shape factor with $\mathrm{k}=0.3$. Whereas, spherical diameter of particles, $d_{p}$, was assumed as the aperture size from passing particles through the sieves (undersize).

Data from Table 2, with Reynold numbers, Re value range within $0.05-247.70$ with particle size between $37-850 \mu \mathrm{m}$, respectively. This explained that Barium Hexaferrite particle mixed in toluene medium in streamline condition or laminar flow (theoretically $\mathrm{Re}$ value below 2100 are laminar flow). From Fig. 6, shows that terminal velocity increasing as the particle size get bigger, with fitting linear line R-squared equal to 0.9957 based on Stokes law. It also explained that increasing in terminal velocity thus bulk density value of Barium Hexaferrite reduced due to large compacts flocs, and also the effect of drag force, $\mathrm{F}_{\mathrm{D}}$, during the free fall of particle in the toluene medium as proved in Eq. (6).

Measurement of turbidity has done by using turbidity meter TU-2016 for undersize particle in which the particle diameter was assumed as the sieves aperture size. From Fig. 5, shows the results of turbidity measurement for $400 \mathrm{~s}$ with time interval $10 \mathrm{~s}$ each data point. This graph was also explained that for smaller particle size shows higher 
turbidity compared to larger particle size from 37$850 \mu \mathrm{m}$.

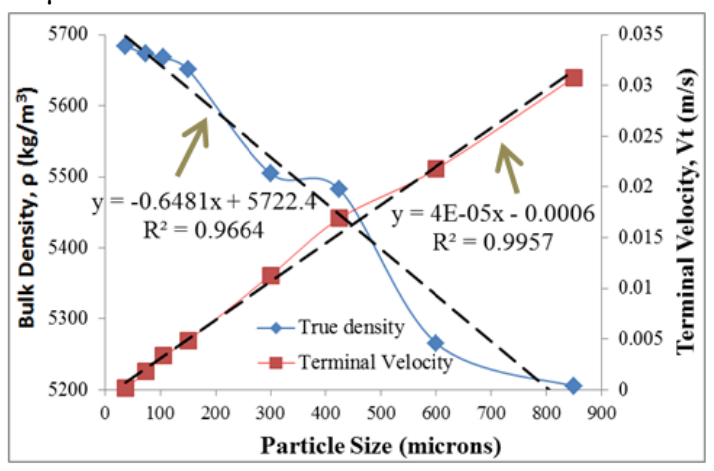

Figure 4. Relationship between bulk density and terminal velocity towards particle size.

Thus, from Fig. 5 it was possible to observe the sedimentation time by plotting the graph of differences between maximum $\left(\mathrm{T}_{\max }\right)$ and minimum $\left(\mathrm{T}_{\min }\right)$ turbidity $\left(\Delta\right.$ Turbidity $\left.=\mathrm{T}_{\max }-\mathrm{T}_{\min }\right)$ of each particle size as shown in Fig. 6.

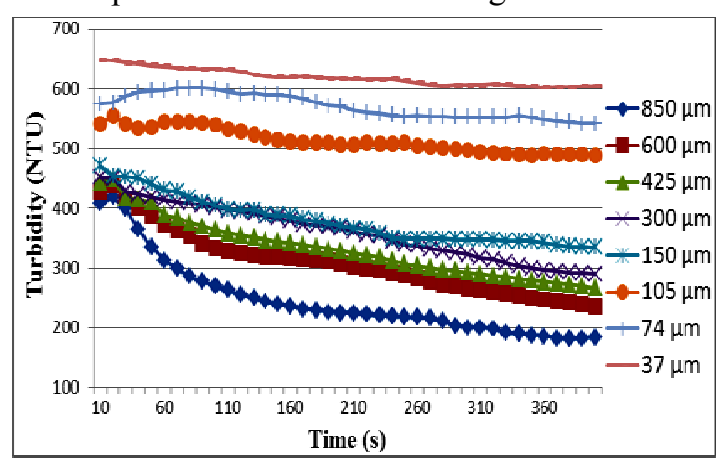

Figure 5. Turbidity (NTU) depends on particle size for 400s.

From Fig. 6 shows the relationship between $\Delta$ Turbidity with particle size, as particle size get larger and $\Delta$ Turbidity increase in value from 47 up to 238 NTU. Graph $\Delta$ Turbidity against particle size, can be divided into two regions (divided region show in the dot line) due to rapidly change in $\Delta$ Turbidity occurred from 150 to $105 \mu \mathrm{m}$. First region from particle size range within $150-$ $850 \mu \mathrm{m}$ yields $\Delta$ Turbidity from 137 up to 238 NTU, respectively, with linear fitting line of experimental data R-squared equal to 0.9955 . Second region shows particle size range within 37$105 \mu \mathrm{m}$ and $\Delta$ Turbidity from 47-66 NTU, respectively, with fitting linear line value, Rsquared equal to 0.9984 . These phenomena could also explain the sedimentation effect occurred more rapidly in first region compared to second region with difference in $\Delta$ Turbidity value are 101 and 19 , respectively. This argument of experimental data was approximately agreed with mathematical model for terminal velocity using stokes law, where particle size range within $300-850 \mu \mathrm{m}$ the free fall or sedimentation occurred rapidly with terminal velocity from 11.28 up to $30.79\left(\times 10^{-3} \mathrm{~m} / \mathrm{s}\right)$ whereas $\Delta$ Turbidity occurred within particle size range 150 $850 \mu \mathrm{m}$.

Thus in Fig. 8, which divided into two regions can be used as the estimation of sedimentation based on size range within two different regions. For particle size range from 150$850 \mu \mathrm{m}$ and $37-105 \mu \mathrm{m}$ can be used linear regression equation, $\mathrm{y}=0.1425 \mathrm{x}+118.54$ and $\mathrm{y}=0.28 \mathrm{x}+36.841$, respectively, due to perfectly fitting linear line.

Additionally, in Fig. 6 shows that higher value of bulk density yields lower value of $\Delta$ Turbidity, which shows that large compact flocs (larger particle size) have a high settling rate that results in low turbidity during settlement.

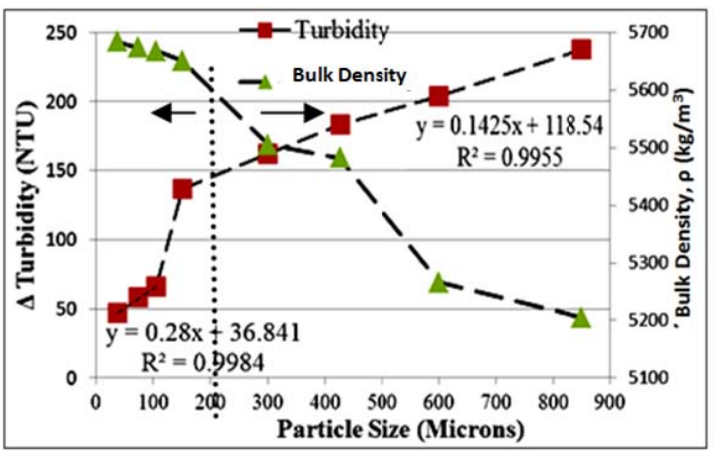

Figure 6. Relationship $\Delta$ Turbidity with bulk density against particle size (flocs).

\section{CONCLUSSIONS}

Particle Size distribution by using Rosin-Rammler mathematical model distribution has been performed with perfectly fitting linear line Rsquared equal to 1 , where distribution function and density function. Reynold numbers for Barium Hexaferrite sample was shown to be laminar flow $(\operatorname{Re}<2100)$ in ethanol medium, whereas terminal velocity value increases as larger particle size, however, bulk density reduce due to contained highly porous in the sample which yields lower bulk density. Turbidity measurement has been performed, as larger particle size yields lower value of turbidity, meanwhile the differences in turbidity for each sample size divided into two regions. This possibly occurred due to higher particle size could 
results the sedimentation time is faster compared to the smaller particle size (flocs).

\section{REFERENCES}

1. Kim, S.H., et al., Effects of $\mathrm{pH}$ and dosage on pollutant removal and floc structure during coagulation. Journal of Microchemical, 2001. p. 197-203.

2. Rebhun, M. \& Lurie, M., Control of organic matter by coagulation and floc separation. Water Science Technology, 1993. 27(11): p. 120.

3. Wilen, B.M., et al., Impacts of structural characteristics on activated sludge floc stability. Water Resource, 2003. 37: p. 3632-3645

4. Bushell, G.C., et al., On techniques for the measurement of the mass fractal dimension of aggregates. Advanced Colloid Interface Science, 2002. 95: p. 1-50

5. Dierickx, D., et al., Statistical extreme value modeling of particle size distributions: experimental grain size distribution type estimation and parameterization of sintered zirconia. Material Characteristic, 2000. 45: p. $61-70$ d.

6. Ramakrishnan, K.N., Investigation of the effect of powder particle size distribution on the powder microstructure and mechanical properties of consolidated material made from a rapidly solidified $\mathrm{Al}, \mathrm{Fe}$, Ce alloy powder: Part I. Powder microstructure.Material Characteristic, 1994. 33: p. 119-28.

7. Garc1'a,.A.M., et al., Application of the RosinRammler and Gates-Gaudin-Schuhmann models to the particle size distribution analysis of agglomerated cork. Materials Characterization, 2004. 52: p. 159- 164

8. Mac1'as, A,D.P.A., et al., Bending strength of black and composite agglomerates of cork. Material Letters, 2003. 57: p. 4004-4008.

9. Ballester A., Verdeja L.F., Sancho J.P., 2000. Metalurgia Extrativa. Madrid, Spain, Síntesis.

10. Tang, P., Greenwood, J., and Raper, J.A., A model to describe the settling behaviour of fractal objects. Journal of Colloids and Interface Science, 2002. 247: p. 210-219.

11. Peter. J., et al., Measuring floc structural characteristics. Reviews in Environmental Science and Bio/Technology, 2005. 4: p. 1-18.

12. Vítěz, T., and Trávníček, P. Particle size distribution of sawdust and wood shavings Mixtures. Vol. 56, 2010, No. 4: p. 154-158.

13. Coulson, J.M., et al., Chemical Engineering, Vol. 2, Particle Technology and Separation Processes, 4th edition, 1999, Butterworth Heinem 